\title{
Review Article \\ Epigenetic Control of Haematopoietic Stem Cell Aging and Its Clinical Implications
}

\author{
Fizzah Aziz Choudry ${ }^{1,2}$ and Mattia Frontini ${ }^{1,2,3}$ \\ ${ }^{1}$ Department of Haematology, University of Cambridge, Cambridge CB2 OPT, UK \\ ${ }^{2}$ National Health Service Blood and Transplant, Cambridge Biomedical Campus, Cambridge CB2 OPT, UK \\ ${ }^{3}$ British Heart Foundation Centre of Excellence, University of Cambridge, Cambridge CB2 OQQ, UK \\ Correspondence should be addressed to Mattia Frontini; mf471@cam.ac.uk
}

Received 17 April 2015; Accepted 1 July 2015

Academic Editor: Lucia Latella

Copyright (C) 2016 F. A. Choudry and M. Frontini. This is an open access article distributed under the Creative Commons Attribution License, which permits unrestricted use, distribution, and reproduction in any medium, provided the original work is properly cited.

Aging, chronic inflammation, and environmental insults play an important role in a number of disease processes through alterations of the epigenome. In this review we explore how age-related changes in the epigenetic landscape can affect heterogeneity within the haematopoietic stem cell (HSC) compartment and the deriving clinical implications.

\section{Introduction}

Aging is associated with alterations in the heterogeneous haematopoietic stem cell (HSC) compartment including changes in clonal composition and lineage contribution. Recent data shows that these changes in functional potential within the HSC population may be modulated by a drift in the epigenome that occurs with increasing age and may ultimately lead to a transcriptional change within the HSC pool. Here we describe the current state of knowledge of haematopoietic stem cell heterogeneity and its changes with age, discuss the evidence for changes in the epigenetic landscape as a potential driver, and propose a model by which these changes may explain some of the pathological consequences of aging.

\section{Haematopoietic Stem Cell Heterogeneity}

The haematopoietic system relies on a small population of HSCs resident in the bone marrow to generate $\sim 10^{11}$ new cells every day. HSCs have the capacity of self-renewal and differentiation through a cascade of progressively committed and lineage restricted progenitors to ultimately generate all mature circulating myeloid and lymphoid cell types [1]. Originally it was thought that HSCs were a single homogenous cell population with the same proliferation and multipotent differentiation capability [2]. However in the last 10 years it has become clear that the HSC compartment is in fact made up of a number of subsets each distinguished by its own selfrenewal capacity (long-term and short-term HSCs) [3-5] and lineage differentiation potential [6].

The first evidence of HSC compartment heterogeneity came from mouse spleen CFU assays; these showed a high degree of variability in numbers and types of colonies produced, challenging the idea of a single homogenous population. However, direct evidence on HSC heterogeneity came from methods that allow assessment of mature cell outputs from limiting numbers and even single HSCs [7-10]. These include the ability of purified single HSCs to repopulate a secondary myeloablated host and cellular barcoding whereby lentiviral gene transfer is used to uniquely label individual HSCs allowing their progenies to be tracked within the transplanted host. This work has led to HSC subsets being distinguished according to their mature cell output. The existence of analogous HSC subsets in humans has been suggested by evidence from therapeutic transplantation in the setting of $\beta$-thalassemia [11].

Although currently defined HSC subsets are able to produce all the mature cell progeny, the ratio of myeloid to lymphoid progeny varies markedly. HSC compartment 
TABLE 1: Currently defined HSC subsets in mouse and their definition by mature cell output and cell surface markers.

\begin{tabular}{lll}
\hline HSC subset & $\begin{array}{l}\text { Predominant mature cell } \\
\text { population }\end{array}$ & $\begin{array}{l}\text { Cell surface markers that have been used for } \\
\text { prospective identification }\end{array}$ \\
\hline $\begin{array}{l}\text { Mouse HSC } \\
\begin{array}{l}\text { Myeloid-biased/lymphoid-deficient } \\
(\alpha)\end{array}\end{array}$ & $\begin{array}{l}\mathrm{Lin}^{-} \mathrm{Sca}^{+} \mathrm{CKit}^{+} \mathrm{CD} 150^{+} \mathrm{CD} 48^{-} \mathrm{CD} 34^{-} \\
\text {Lymphoid-biased/myeloid-deficient } \\
(\gamma / \delta)\end{array}$ & $+\mathrm{CD} 150^{\mathrm{High}}, \mathrm{CD}^{-} 1^{+}$ \\
$\begin{array}{l}\text { Balanced }(\beta) \\
\text { Platelet-biased }\end{array}$ & Lymphoid & $+\mathrm{CD} 229^{+}$ \\
Human HSC & $\begin{array}{l}\text { Myeloid and lymphoid } \\
\text { Platelet and myeloid }\end{array}$ & $+\mathrm{VWF}^{+}, \mathrm{CD}^{+} 1^{+}$ \\
\hline
\end{tabular}

Lin: lineage markers; VWF: von Willebrand factor.

subsets have been described as myeloid-biased, lymphoidbiased, or balanced $[3,12]$ or by others as lymphoiddeficient $(\alpha)$, myeloid-deficient $(\gamma$ and $\delta$ ), and balanced $(\beta)$, respectively [13] (Table 1). While the majority of accumulated evidence comes from mouse models there is also support for myeloid-biased and lymphoid-biased HSC subsets in humans $[14,15]$. While serial transplantation experiments are able to define HSC subsets by their progeny of mature cells in vivo there is not yet an effective method to prospectively distinguish HSC subsets at molecular level. An attempt to prospectively enrich the myeloid- and lymphoid-biased subsets has been made by defining these populations based on CD150 cell surface level. Myeloid-biased HSC subsets express higher levels of this surface marker compared to lymphoidbiased HSCs [16-18]. However, this is not considered an ideal marker as the cell populations separated are not pure and expression of CD150 changes when cells are manipulated and transplanted $[19,20]$. Recently lymphoid-biased HSCs have been shown to have higher expression of the surface marker CD229 [21].

A further HSC subset recently identified is the megakaryocytic or platelet-biased HSC subset (Table 1). This has been prospectively defined by reporter gene expression and/or surface markers that are highly expressed in the megakaryocyte/platelet lineage: VWF [22] and CD41 (ITGA2B) [23]. Approximately $60 \%$ of mouse HSCs have been shown to coexpress VWF and when these are serially transplanted in limiting numbers they effect highly platelet-biased reconstitution [22]. Interestingly, this population also has a strong myeloid lineage bias whereas VWF-HSC contribution to the myeloid lineage is minimal [22]. It has therefore been suggested that previous studies that identified a myeloid-biased HSC subset $[3,12,13]$ may have identified both plateletbiased and myeloid-biased HSCs in the absence of methods to evaluate platelet output. Furthermore, the platelet-biased HSC subset has been hierarchically placed at the apex of the haematopoietic tree, due to its ability to give rise to the lymphoid-biased HSC subset [22]. Similarly, a platelet- and myeloid-biased HSC subset has been identified by the expression of the megakaryocyte/platelet cell surface marker and part of the glycoprotein IIb/IIIa fibrinogen receptor: CD41, a population that may possibly be phenotypically analogous to that expressing VWF [23]. CD41 had been known to be expressed in embryonic HSCs but then switched off after birth [24-27]. Its expression has now been demonstrated on a subset of mouse HSCs which show a platelet and myeloid bias on serial transplantation with a knockdown of CD41 resulting in reduced levels of all mature blood cell lineages [23]. A platelet-biased HSC subset has not yet been defined in the human.

While the majority of evidence on HSC lineage commitment to date, including that presented here, derives from transplantation studies, transplantation creates an artificial environment that is limited by engraftment-associated inflammation. The use of novel in situ inducible labelling techniques has enabled the study of physiological haematopoiesis in a healthy bone marrow environment. Two recent mouse studies using this approach have proposed a model of haematopoiesis that while supporting data from transplantation studies suggest that classical long-term HSCs have a limited contribution to steady-state haematopoiesis [28, 29]. Rather HSC heterogeneity is produced by thousands of multipotent clones within a reservoir of cells traditionally defined as short-term HSCs and multipotent progenitors [29], which are shown to be longer-lived than previously thought and have considerable self-renewal capability. These novel techniques while still in their early stages are anticipated to provide further insight into haematopoietic lineage commitment in more physiological conditions.

\section{Aging within the HSC Compartment}

Multiple studies have established that aging, both in mouse and in human, leads to a myeloid-skewed haematopoietic system, with diminished representation of lymphoid cell populations and an increased representation of myeloid progenitors that has been shown to be associated with a myeloidbiased HSC population [17, 30-32]. Furthermore, it has been shown that serial transplantation of young mouse HSCs into young secondary hosts selectively expands a myeloid-biased HSC population independent of a nonaging microenvironment which suggests that HSC lineage bias is intrinsic to the cell itself [32]. In addition, there is also evidence in the mouse of an age-related increase in platelet-biased HSCs 
defined by their expression of CD41. When transplanted these cells show a predominantly platelet- and myeloidbiased reconstitution, which suggests that the myeloid-biased HSC population seen in elderly mice may also be plateletbiased [23]. Although age-related changes in VWF+ HSCs have not been evaluated it could be speculated that this population would also expand with age since this subset leads to a platelet- and myeloid-biased reconstitution. This is supported by similarities in gene expression profiles between VWF+ HSCs and aged HSCs, both demonstrating significant upregulation of megakaryocyte-lineage genes such as Selp and $\mathrm{Clu}$ as well as the upregulation of VWF in aged HSCs [33]. Expansion of the VWF+ HSC population with age does not, however, support the hierarchical positioning of this population above the lymphoid-biased HSC subset as this population is known to decrease with age.

In humans, age-related haematopoietic changes include decreased bone marrow cellularity [34], attenuated lymphoid potential [35], increased incidence of myeloproliferative disorders and myeloid malignancies [36], and increased incidence of thrombosis [37]. As in mouse, these findings have been correlated with an accumulation of HSCs within the aged human bone marrow, which, while being able to generate both lymphoid and myeloid progeny in culture and in xenotransplant, showed significant myeloid skewing compared with young HSCs [14]. The existence of an agerelated platelet-biased HSC subset in humans has not yet been investigated.

Postulated mechanisms that may lead to a decline in lymphoid differentiation with age include a gradual erosion of lymphopoietic potential within the HSC compartment over time, a conversion of lymphoid-biased to myeloid-biased HSCs, and a gradual dominance of myeloid-biased HSCs either due to their slower turnover leading to increased survival or a higher self-renewal capacity leading to clonal dominance with time $[12,38]$. How these changes are controlled and regulated is still unclear. Aging within the HSC compartment has been shown to be associated with decreased functionality due to elevated levels of reactive oxygen species [39] and accumulation of DNA damage [40] which may account for some of the differences observed with age. It is clear, however, that these mechanisms do not account for all of the cellular and molecular attributes that are associated with aging of the HSC compartment, indicating that other mechanisms must be involved. There are a number of lines of evidence showing that HSC aging is transcriptionally regulated with differences in gene expression between young and aged HSC populations [14]. This suggests that alteration in gene expression by changes in the epigenetic landscape may play a key role in modulating age-related changes in the HSC compartment.

\section{Epigenetic Regulation of HSC Aging}

The term epigenetic encompasses all heritable changes in gene expression that are not due to changes in DNA sequence. These are modifications of the genome or of DNA-associated proteins. They include changes in DNA methylation, histone modifications, and changes in chromatin structure that impact on the accessibility of genetic loci for transcription machinery. Noncoding RNAs also play a critical role in epigenetic regulation. It is because of epigenetic regulation that a cell retains its identity and its gene expression profile through cell division and differentiation without altering its DNA sequence. However, epigenetic marks can also change over time [41-43] due to aging and environment, which may be related to mutations in epigenetic regulators, although the underlying molecular mechanisms are still unclear. It is this change in the epigenetic landscape of the HSC compartment that has been suggested to lead to age-related changes.

While the coding potential of the genome lies in the arrangement of the four nucleotides, additional information affecting phenotype is stored in the distribution of methylated cytosine (5-methylcytosine). DNA methylation occurs at CpG motifs that are interspersed within the genome in clusters called CpG islands. Densely methylated promoter regions are associated with compacted chromatin structure and therefore transcriptional shutdown; conversely demethylation leads to chromatin opening and therefore gene expression.

Aging in somatic tissues has been associated with global hypomethylation [44] where the majority of cells are postmitotic. In contrast in aged HSCs, which are characterised by mitotic potential often longer than the organism lifespan, a significant degree of global DNA hypermethylation is observed. However, if HSCs are taken to the end of their proliferation potential by serial transplantation, although not a physiological condition, similar patterns of global hypomethylation are observed [33, 45]. Young HSCs gain DNA methylation in regions associated with nonhaematopoietic lineages and significant losses of DNA methylation in genomic regions associated with blood cell production. Conversely, aged HSCs display gains of DNA methylation in genomic regions associated with lymphoid and erythroid lineages; both lineages decline in number during aging. Interestingly, the majority of genes differentially methylated during HSC aging were associated with lineage potential and highly expressed downstream of the HSC in the haematopoietic tree [45]. Furthermore, age-related hypermethylated regions were enriched for targets of the Polycomb group of proteins, known to establish repressive chromatin [33, 45].

Regulators of DNA methylation include DNA methyltransferases (DNMTs) that drive methylation of CpG motifs and the ten-eleven translocation (Tet) enzymes that regulate demethylation. Functional studies implicate these epigenetic regulators in the aging process within the HSC compartment. Genetic alteration studies demonstrate that DNMT1 is responsible for maintaining methylation and its loss in the HSC compartment leads to myeloid skewing and selfrenewal defects [46, 47]. Furthermore, loss of both DNMT3A and DNMT3B leads to a severe arrest in HSC differentiation [48]. Loss of Tet2 in mice attenuates differentiation and leads to myeloid transformation and myeloid malignancies [4951]. Somatic mutations in Tet 2 have also been shown in normal elderly human subjects [52]. Importantly, there is now 
evidence of differential expression of both DNMT and Tet2 enzymes in aged HSCs compared with young HSCs [33, 45].

Histone posttranslational modifications including acetylation, methylation, phosphorylation, sumoylation, and ubiquitination can change chromatin structure and therefore DNA accessibility to transcriptional machinery. These modifications may act separately or synergistically to regulate gene expression. Priming DNA in such a way precedes lineage commitment in the HSC population as seen by histone modifications associated with gene expression in committed mature cell populations already present within the HSC compartment $[53,54]$. This observation is similar to that of differential DNA methylation of genes that are expressed downstream described above. HSCs also utilise Polycomb (PcG) genes to regulate aging, prevent premature aging, and maintain HSC function by forming PcG repressive complexes (PRC). While the PRC1 complex possesses H2AK119 ubiquitin ligase activity, PRC2 acts as a H3K27 methyltransferase.

Age-related changes in histone modifications also provide mechanisms that may contribute to changes seen in the aged HSC compartment. Aged HSCs show methylation of H3K4me3, a mark of active chromatin, which correlate with increases in gene expression of HSC identity and self-renewal genes [33]. Differential methylation of the repressive mark H3K27me3 has also been shown in aged HSCs, with increased $\mathrm{H} 3 \mathrm{~K} 27 \mathrm{me} 3$ on a number of promoters [33]. HSC aging is also associated with low levels of H4K16ac activation mark [55]. Furthermore, interdependency between DNA methylation and histone modification exists and it might be relevant to HSC aging; however its full understanding is reliant on the development of assays that require smaller cell numbers to detect both epigenetic marks in the same samples.

Functional studies, where lysine-specific demethylases that drive $\mathrm{H} 3 \mathrm{~K} 4$ demethylation and regulate chromatin accessibility have been genetically modulated, show their critical role in stem cell differentiation. $\mathrm{Kdm} 3 \mathrm{a}$ and $\mathrm{Kdm} 5 \mathrm{a}$ have also been implicated in regulating stem cell aging, a notion supported by the fact that these proteins' expression decreases with age $[33,56,57]$. Knockdown of the lysine demethylase Kdm5b (Jarid1b) leads to increased HSC activity [58] and is also known to be differentially expressed with aging [45]. Knockout studies of the H3K27me3 demethylase Kdm6a (UTX1) have shown it to be a key regulator of haematopoiesis [59] and knockdown in C. elegans extends their lifespan [60]. Furthermore, HSCs deficient in the Bmil component of PRC1 [61-63] as well as the Ezh1 [64] and Eed [65] components of PRC2 show a severe defect with marked derepression of the tumour suppressor and aging-associated complex Ink4a/Arf.

Noncoding RNAs are RNAs that are not translated into protein but are known to play an important epigenetic regulatory role. While the direct impact of noncoding RNAs on $\mathrm{HSC}$ aging requires further investigation, there is evidence to suggest that noncoding RNAs are both highly expressed and regulate HSC survival and function. The microRNA miR$125 \mathrm{~b}$ is highly expressed in HSCs and plays a role in regulating survival $[66,67]$ whereas miR-126 knockdown is associated with a myeloid-biased HSC compartment [68]. The long noncoding RNA Xist is essential for HSC survival [69].

\section{Consequences of Age-Related Changes in the HSC Epigenetic Landscape}

It is not clear if there is a physiological benefit of a progressive myeloid bias within the HSC compartment. However, it is clear that the epigenetic drift that leads to this phenotype correlates with the increased incidence with age of myeloproliferative disorders and myeloid malignancies as well as increased risk of infection and thrombosis (Figure 1).

Myeloproliferative disorders, myelodysplastic syndromes, and haematological malignancies [36] are attributed to accumulation of mutations in the aging HSC compartment, many of which are involved in epigenetic regulation of the HSC population such as Tet2 and DNMT3 [52, 70-73]. DNA methylation plays an important role in the pathogenesis and progression of myelodysplastic syndromes where DNA hypermethylation and methyl silencing are implicated as the pivotal mechanism [74-76]. In accordance with this, clinical trials for agents that inhibit DNA methylation are ongoing in myelodysplastic syndromes [77-80]. Acute myeloid leukaemia is typically associated with hypomethylation caused by deregulation of DNMT1 or possibly overexpression of Tet family genes [81, 82].

The myeloid skewing that occurs with advancing age may also be associated with the decline in adaptive immune response with a resultant increased risk of infection that confers high levels of morbidity and mortality in the elderly population. There is a known reduction in numbers of circulating naïve lymphocytes that could be a direct effect of a changing epigenetic landscape within the HSC compartment leading to a reduced mature lymphoid lineage generation [35, 83-85].

Age is also an important risk factor for coronary and cerebrovascular platelet thrombosis [37], conditions that have been causally linked to increased platelet activity and increased platelet mass [86]. The setting of acute arterial thrombosis has been likened to states of increased haemostatic demand. While only correlative and chronological data exists, it is reasonable to hypothesise that as in states of increased haemostatic demand, when functional circulating platelet mass drops, there is a consequential regulation of megakaryocyte activity and platelet production. The nature of this control system and how megakaryocyte activity is regulated is still unknown; however, increases in platelet mass have been correlated with increased megakaryocyte ploidy [87-89]. It is also possible that this feedback may be at the level of the HSC by an expansion of a platelet-biased HSC subset. With an age-related increase in both platelet mass [90, 91] and platelet-biased HSCs [23] it could be suggested that the recently discovered megakaryocyte- or platelet-primed HSC subset may have thrombotic implications in the elderly. It is reasonable to hypothesise that age-related changes in the epigenetic landscape with age lead to a platelet-biased HSC subset that is primed to generate a transcriptionally distinct megakaryocyte phenotype due to defined epigenetic marks that leads to increased platelet mass and activity. In support of this increasing megakaryocyte ploidy is associated with significantly increased expression of VWF and CD41 [92], both markers of a platelet-biased HSC subset. 

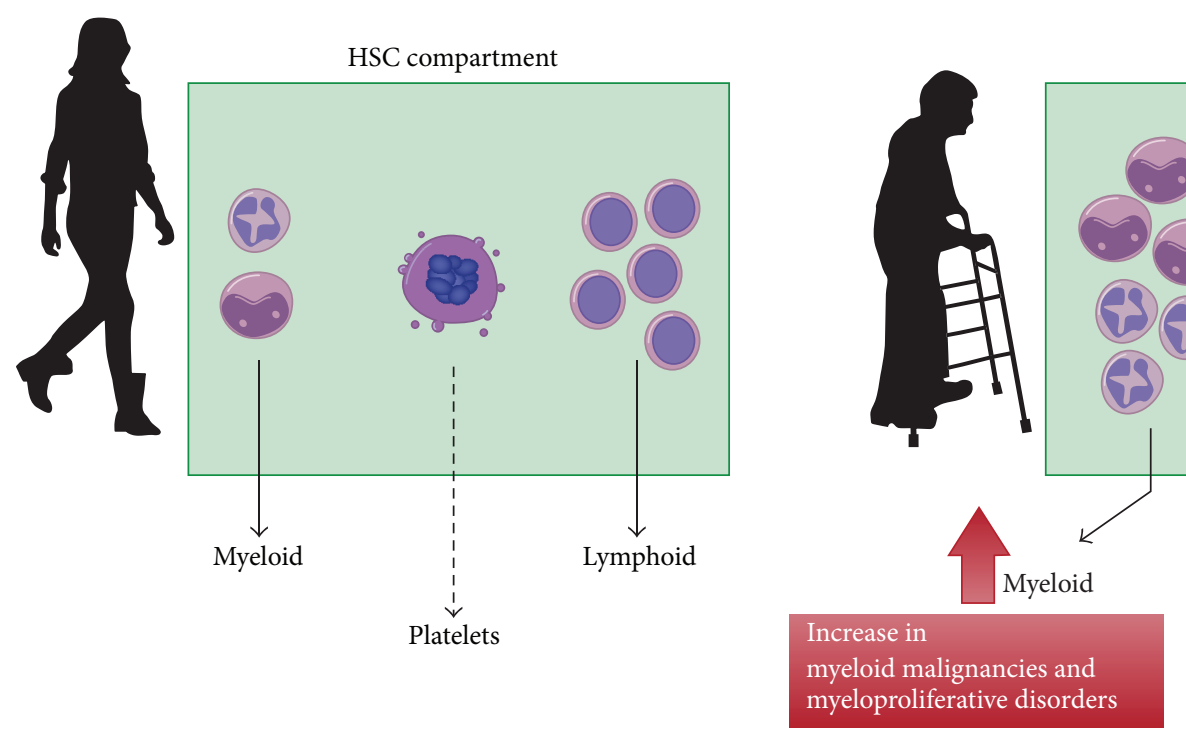

HSC compartment

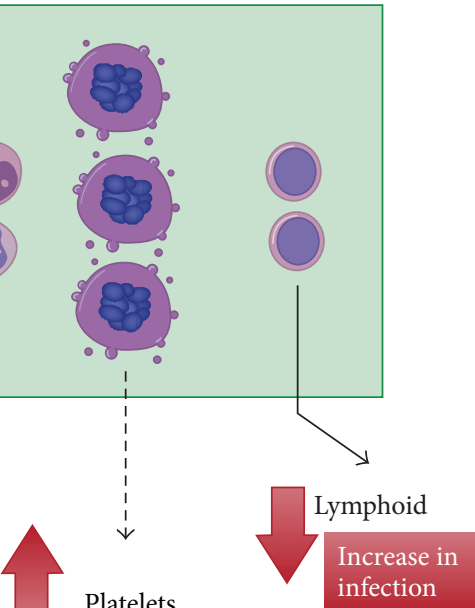

Increase in

arterial thrombosis

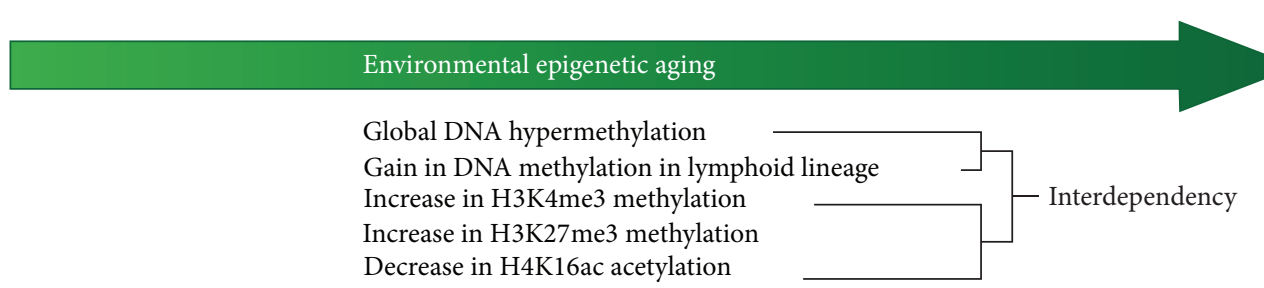

FIGURE 1: Drift in the epigenetic landscape that occurs with environmental and biological factors associated with aging leads to transcriptional differences between the HSC compartments in the young population compared with the elderly population. This is proposed to give rise to expansion of particular clones within the heterogeneous HSC pool to produce a myeloid- and platelet-skewed haematopoietic system. These changes may play an important role in driving the increased incidence of myeloproliferative disorders, myeloid malignancies, infection, and acute arterial thrombosis observed in the elderly.

A complex relationship exists between megakaryocytes and HSCs, with a number of phenotypic and molecular similarities including surface markers (CD41 and VWF), thrombopoietin (TPO), its receptor (MPL) and CXCR4, transcription factor dependence (RUNX-1, GATA-2, Evi-1, SCL/TAL1, and Ets family transcription factors), signalling pathways, and proximity within the bone marrow niche [93]. Furthermore, maintenance of both megakaryocytes and HSCs crucially depends on TPO [94-96]. While the functional relevance of these similarities remains unclear there is now a body of evidence that supports the existence of tight homeostatic control mechanisms along the HSC-megakaryocyteplatelet axis. Recent reports demonstrate a critical role for megakaryocytes in maintaining HSC quiescence through either release of CXCL4 [97] and TGF $\beta$ [98] or indeed CD41 expression [23]. In mouse acute depletion of megakaryocytes leads to HSC expansion and proliferation implying a critical regulatory feedback mechanism between megakaryocytes and the HSC compartment [97]. Moreover, platelets may also regulate HSC quiescence through effects on circulating TPO concentrations [99]. Further work is required to gain a full understanding of this complex relationship.

\section{Conclusions}

Epigenetic changes in the HSC compartment lead to the phenotypic and functional changes that are seen in the mature cell output of the haematopoietic system with advancing age. Although these epigenetic changes are not directly pathological they produce an environment that is conducive to pathological processes that are seen in prevalence in the elderly population. In some disease processes age-related epigenetic changes may be more directly pathogenic but in other complex diseases such as coronary artery disease they may indeed account for the missing heritability determinants that have not been accounted for to date by genetic studies of sequence variation [100]. Our understanding of the role of epigenetic changes in stem cell regulation, though quickly expanding, is only at its beginnings. However, as epigenetic marks are potentially reversible this opens up the possibility 
of manipulating epigenetic states and ultimately changing the way the genome functions.

\section{Conflict of Interests}

The authors declare that there is no conflict of interests regarding the publication of this paper.

\section{Acknowledgments}

Fizzah Aziz Choudry is recipient of an MRC clinical fellowship (MR/K024043/1). Mattia Frontini is supported by the BHF Cambridge Centre of Excellence [RE/13/6/30180] and EU-FP7 project BLUEPRINT (282510).

\section{References}

[1] D. Bryder, D. J. Rossi, and I. L. Weissman, "Hematopoietic stem cells: the paradigmatic tissue-specific stem cell," The American Journal of Pathology, vol. 169, no. 2, pp. 338-346, 2006.

[2] N. M. Blackett, E. Necas, and E. Frindel, "Diversity of haematopoietic stem cell growth from a uniform population of cells," Nature, vol. 322, no. 6076, pp. 289-290, 1986.

[3] C. E. Muller-Sieburg, R. H. Cho, L. Karlsson, J.-F. Huang, and H. B. Sieburg, "Myeloid-biased hematopoietic stem cells have extensive self-renewal capacity but generate diminished lymphoid progeny with impaired IL-7 responsiveness," Blood, vol. 103, no. 11, pp. 4111-4118, 2004.

[4] T. Reya, S. J. Morrison, M. F. Clarke, and I. L. Weissman, "Stem cells, cancer, and cancer stem cells," Nature, vol. 414, no. 6859, pp. 105-111, 2001.

[5] I. L. Weissman, D. J. Anderson, and F. Gage, "Stem and progenitor cells: origins, phenotypes, lineage commitments, and transdifferentiations," Annual Review of Cell and Developmental Biology, vol. 17, pp. 387-403, 2001.

[6] C. E. Müller-Sieburg, R. H. Cho, M. Thoman, B. Adkins, and H. B. Sieburg, "Deterministic regulation of hematopoietic stem cell self-renewal and differentiation," Blood, vol. 100, no. 4, pp. 1302-1309, 2002.

[7] M. J. Kiel, Ö. H. Yilmaz, T. Iwashita, O. H. Yilmaz, C. Terhorst, and S. J. Morrison, "SLAM family receptors distinguish hematopoietic stem and progenitor cells and reveal endothelial niches for stem cells," Cell, vol. 121, no. 7, pp. 1109-1121, 2005.

[8] M. Osawa, K.-I. Hanada, H. Hamada, and H. Nakauchi, "Longterm lymphohematopoietic reconstitution by a single CD34low/negative hematopoietic stem cell," Science, vol. 273, no. 5272, pp. 242-245, 1996.

[9] R. Lu, N. F. Neff, S. R. Quake, and I. L. Weissman, “Tracking single hematopoietic stem cells in vivo using high-throughput sequencing in conjunction with viral genetic barcoding," Nature Biotechnology, vol. 29, no. 10, pp. 928-934, 2011.

[10] A. Gerrits, B. Dykstra, O. J. Kalmykowa et al., "Cellular barcoding tool for clonal analysis in the hematopoietic system," Blood, vol. 115, no. 13, pp. 2610-2618, 2010.

[11] M. Cavazzana-Calvo, E. Payen, O. Negre et al., "Transfusion independence and HMGA2 activation after gene therapy of human $\beta$-thalassaemia," Nature, vol. 467, no. 7313, pp. 318-322, 2010.

[12] C. E. Muller-Sieburg, H. B. Sieburg, J. M. Bernitz, and G. Cattarossi, "Stem cell heterogeneity: implications for aging and regenerative medicine," Blood, vol. 119, no. 17, pp. 3900-3907, 2012.

[13] B. Dykstra, D. Kent, M. Bowie et al., "Long-term propagation of distinct hematopoietic differentiation programs in vivo," Cell Stem Cell, vol. 1, no. 2, pp. 218-229, 2007.

[14] W. W. Pang, E. A. Price, D. Sahoo et al., "Human bone marrow hematopoietic stem cells are increased in frequency and myeloid-biased with age," Proceedings of the National Academy of Sciences of the United States of America, vol. 108, no. 50, pp. 20012-20017, 2011.

[15] M. Cavazzana-Calvo, A. Fischer, F. D. Bushman, E. Payen, S. Hacein-Bey-Abina, and P. Leboulch, "Is normal hematopoiesis maintained solely by long-term multipotent stem cells?” Blood, vol. 117, no. 17, pp. 4420-4424, 2011.

[16] G. A. Challen, N. C. Boles, S. M. Chambers, and M. A. Goodell, "Distinct hematopoietic stem cell subtypes are differentially regulated by TGF- $\beta 1$," Cell Stem Cell, vol. 6, no. 3, pp. 265-278, 2010.

[17] I. Beerman, D. Bhattacharya, S. Zandi et al., "Functionally distinct hematopoietic stem cells modulate hematopoietic lineage potential during aging by a mechanism of clonal expansion," Proceedings of the National Academy of Sciences of the United States of America, vol. 107, no. 12, pp. 5465-5470, 2010.

[18] Y. Morita, H. Ema, and H. Nakauchi, "Heterogeneity and hierarchy within the most primitive hematopoietic stem cell compartment," Journal of Experimental Medicine, vol. 207, no. 6, pp. 1173-1182, 2010.

[19] G. J. Spangrude, D. M. Brooks, and D. B. Tumas, "Longterm repopulation of irradiated mice with limiting numbers of purified hematopoietic stem cells: in vivo expansion of stem cell phenotype but not function," Blood, vol. 85, no. 4, pp. 1006-1016, 1995.

[20] T. D. Randall and I. L. Weissman, "Phenotypic and functional changes induced at the clonal level in hematopoietic stem cells after 5-fluorouracil treatment," Blood, vol. 89, no. 10, pp. 35963606, 1997.

[21] H. Oguro, L. Ding, and S. J. Morrison, "SLAM family markers resolve functionally distinct subpopulations of hematopoietic stem cells and multipotent progenitors," Cell Stem Cell, vol. 13, no. 1, pp. 102-116, 2013.

[22] A. Sanjuan-Pla, I. C. Macaulay, C. T. Jensen et al., "Plateletbiased stem cells reside at the apex of the haematopoietic stemcell hierarchy," Nature, vol. 502, no. 7470, pp. 232-236, 2013.

[23] C. Gekas and T. Graf, "CD41 expression marks myeloid-biased adult hematopoietic stem cells and increases with age," Blood, vol. 121, no. 22, pp. 4463-4472, 2013.

[24] M. J. Ferkowicz, M. Starr, X. Xie et al., "CD41 expression defines the onset of primitive and definitive hematopoiesis in the murine embryo," Development, vol. 130, no. 18, pp. 43934403, 2003.

[25] K. E. Rhodes, C. Gekas, Y. Wang et al., "The emergence of hematopoietic stem cells is initiated in the placental vasculature in the absence of circulation," Cell Stem Cell, vol. 2, no. 3, pp. 252-263, 2008.

[26] C. Robin, K. Ottersbach, J.-C. Boisset, A. Oziemlak, and E. Dzierzak, "CD41 is developmentally regulated and differentially expressed on mouse hematopoietic stem cells," Blood, vol. 117, no. 19, pp. 5088-5091, 2011.

[27] N. Debili, C. Robin, V. Schiavon et al., "Different expression of CD41 on human lymphoid and myeloid progenitors from adults and neonates," Blood, vol. 97, no. 7, pp. 2023-2030, 2001. 
[28] K. Busch, K. Klapproth, M. Barile et al., "Fundamental properties of unperturbed haematopoiesis from stem cells in vivo," Nature, vol. 518, no. 7540, pp. 542-546, 2015.

[29] J. Sun, A. Ramos, B. Chapman et al., "Clonal dynamics of native haematopoiesis," Nature, vol. 514, no. 7522, pp. 322-327, 2014.

[30] C. Benz, M. R. Copley, D. G. Kent et al., "Hematopoietic stem cell subtypes expand differentially during development and display distinct lymphopoietic programs," Cell Stem Cell, vol. 10, no. 3, pp. 273-283, 2012.

[31] R. H. Cho, H. B. Sieburg, and C. E. Muller-Sieburg, "A new mechanism for the aging of hematopoietic stem cells: aging changes the clonal composition of the stem cell compartment but not individual stem cells," Blood, vol. 111, no. 12, pp. 55535561, 2008.

[32] B. Dykstra, S. Olthof, J. Schreuder, M. Ritsema, and G. D. Haan, "Clonal analysis reveals multiple functional defects of aged murine hematopoietic stem cells," Journal of Experimental Medicine, vol. 208, no. 13, pp. 2691-2703, 2011.

[33] D. Sun, M. Luo, M. Jeong et al., "Epigenomic profiling of young and aged HSCs reveals concerted changes during aging that reinforce self-renewal," Cell Stem Cell, vol. 14, no. 5, pp. 673-688, 2014.

[34] T. Ogawa, M. Kitagawa, and K. Hirokawa, "Age-related changes of human bone marrow: a histometric estimation of proliferative cells, apoptotic cells, T cells, B cells and macrophages," Mechanisms of Ageing and Development, vol. 117, no. 1-3, pp. 5768, 2000.

[35] P. J. Linton and K. Dorshkind, "Age-related changes in lymphocyte development and function," Nature Immunology, vol. 5, no. 2, pp. 133-139, 2004.

[36] M. A. Lichtman and J. M. Rowe, "The relationship of patient age to the pathobiology of the clonal myeloid diseases," Seminars in Oncology, vol. 31, no. 2, pp. 185-197, 2004.

[37] T. Gordon and W. B. Kannel, "Predisposition to atherosclerosis in the head, heart, and legs. The Framingham study," The Journal of the American Medical Association, vol. 221, no. 7, pp. 661-666, 1972.

[38] M. R. Copley, P. A. Beer, and C. J. Eaves, "Hematopoietic stem cell heterogeneity takes center stage," Cell Stem Cell, vol. 10, no. 6, pp. 690-697, 2012.

[39] K. Ito, A. Hirao, F. Arai et al., "Reactive oxygen species act through p38 MAPK to limit the lifespan of hematopoietic stem cells," Nature Medicine, vol. 12, no. 4, pp. 446-451, 2006.

[40] D. J. Rossi, D. Bryder, J. Seita, A. Nussenzweig, J. Hoeijmakers, and I. L. Weissman, "Deficiencies in DNA damage repair limit the function of haematopoietic stem cells with age," Nature, vol. 447, no. 7145, pp. 725-729, 2007.

[41] J.-P. Issa, "Aging and epigenetic drift: a vicious cycle," Journal of Clinical Investigation, vol. 124, no. 1, pp. 24-29, 2014.

[42] M. F. Fraga and M. Esteller, "Epigenetics and aging: the targets and the marks," Trends in Genetics, vol. 23, no. 8, pp. 413-418, 2007.

[43] S. Han and A. Brunet, "Histone methylation makes its mark on longevity," Trends in Cell Biology, vol. 22, no. 1, pp. 42-49, 2012.

[44] S. Gonzalo, "Epigenetic alterations in aging," Journal of Applied Physiology, vol. 109, no. 2, pp. 586-597, 2010.

[45] I. Beerman, C. Bock, B. S. Garrison et al., "Proliferationdependent alterations of the DNA methylation landscape underlie hematopoietic stem cell aging," Cell Stem Cell, vol. 12, no. 4, pp. 413-425, 2013.
[46] A.-M. Bröske, L. Vockentanz, S. Kharazi et al., "DNA methylation protects hematopoietic stem cell multipotency from myeloerythroid restriction," Nature Genetics, vol. 41, no. 11, pp. 1207-1215, 2009.

[47] J. J. Trowbridge, J. W. Snow, J. Kim, and S. H. Orkin, "DNA methyltransferase 1 is essential for and uniquely regulates hematopoietic stem and progenitor cells," Cell Stem Cell, vol. 5, no. 4, pp. 442-449, 2009.

[48] G. A. Challen, D. Sun, A. Mayle et al., "Dnmt3a and Dnmt3b have overlapping and distinct functions in hematopoietic stem cells," Cell Stem Cell, vol. 15, pp. 350-364, 2014.

[49] M. Ko, H. S. Bandukwala, J. An et al., “Ten-eleven-translocation 2 (TET2) negatively regulates homeostasis and differentiation of hematopoietic stem cells in mice," Proceedings of the National Academy of Sciences of the United States of America, vol. 108, no. 35, pp. 14566-14571, 2011.

[50] Z. Li, X. Cai, C.-L. Cai et al., "Deletion of Tet2 in mice leads to dysregulated hematopoietic stem cells and subsequent development of myeloid malignancies," Blood, vol. 118, no. 17, pp. 4509-4518, 2011.

[51] K. Moran-Crusio, L. Reavie, A. Shih et al., "Tet2 loss leads to increased hematopoietic stem cell self-renewal and myeloid transformation," Cancer Cell, vol. 20, no. 1, pp. 11-24, 2011.

[52] L. Busque, J. P. Patel, M. E. Figueroa et al., "Recurrent somatic TET2 mutations in normal elderly individuals with clonal hematopoiesis," Nature Genetics, vol. 44, no. 11, pp. 1179-1181, 2012.

[53] J. L. Attema, P. Papathanasiou, E. C. Forsberg, J. Xu, S. T. Smale, and I. L. Weissman, "Epigenetic characterization of hematopoietic stem cell differentiation using miniChIP and bisulfite sequencing analysis," Proceedings of the National Academy of Sciences of the United States of America, vol. 104, no. 30, pp. 12371-12376, 2007.

[54] J. Maës, M. Maleszewska, C. Guillemin et al., "Lymphoidaffiliated genes are associated with active histone modifications in human hematopoietic stem cells," Blood, vol. 112, no. 7, pp. 2722-2729, 2008.

[55] M. C. Florian, K. Dörr, A. Niebel et al., "Cdc42 activity regulates hematopoietic stem cell aging and rejuvenation," Cell Stem Cell, vol. 10, no. 5, pp. 520-530, 2012.

[56] M. Herzog, E. Josseaux, S. Dedeurwaerder, E. Calonne, M. Volkmar, and F. Fuks, "The histone demethylase Kdm3a is essential to progression through differentiation," Nucleic Acids Research, vol. 40, no. 15, pp. 7219-7232, 2012.

[57] B. L. Kidder, G. Hu, and K. Zhao, "KDM5B focuses H3K4 methylation near promoters and enhancers during embryonic stem cell self-renewal and differentiation," Genome Biology, vol. 15, article R32, 2014.

[58] S. Cellot, K. J. Hope, J. Chagraoui et al., "RNAi screen identifies Jaridlb as a major regulator of mouse HSC activity," Blood, vol. 122, no. 9, pp. 1545-1555, 2013.

[59] S. Thieme, T. Gyárfás, C. Richter et al., "The histone demethylase UTX regulates stem cell migration and hematopoiesis," Blood, vol. 121, no. 13, pp. 2462-2473, 2013.

[60] T. J. Maures, E. L. Greer, A. G. Hauswirth, and A. Brunet, "The H3K27 demethylase UTX-1 regulates C. elegans lifespan in a germline-independent, insulin-dependent manner," Aging Cell, vol. 10, no. 6, pp. 980-990, 2011.

[61] I.-K. Park, D. Qian, M. Kiel et al., "Bmi-1 is required for maintenance of adult self-renewing haematopoietic stem cells," Nature, vol. 423, no. 6937, pp. 302-305, 2003. 
[62] A. Iwama, H. Oguro, M. Negishi et al., "Enhanced self-renewal of hematopoietic stem cells mediated by the polycomb gene product Bmi-1," Immunity, vol. 21, no. 6, pp. 843-851, 2004.

[63] H. Oguro, A. Iwama, Y. Morita, T. Kamijo, M. Van Lohuizen, and H. Nakauchi, "Differential impact of Ink4a and Arf on hematopoietic stem cells and their bone marrow microenvironment in Bmil-deficient mice," Journal of Experimental Medicine, vol. 203, no. 10, pp. 2247-2253, 2006.

[64] I. Hidalgo, A. Herrera-Merchan, J. M. Ligos et al., "Ezh1 is required for hematopoietic stem cell maintenance and prevents senescence-like cell cycle arrest," Cell Stem Cell, vol. 11, no. 5, pp. 649-662, 2012.

[65] H. Xie, J. Xu, J. H. Hsu et al., "Polycomb repressive complex 2 regulates normal hematopoietic stem cell function in a developmental-stage-specific manner," Cell Stem Cell, vol. 14, no. 1, pp. 68-80, 2014.

[66] S. Guo, J. Lu, R. Schlanger et al., "MicroRNA miR-125a controls hematopoietic stem cell number," Proceedings of the National Academy of Sciences of the United States of America, vol. 107, no. 32, pp. 14229-14234, 2010.

[67] A. G. L. Ooi, D. Sahoo, M. Adorno, Y. Wang, I. L. Weissman, and C. Y. Park, "MicroRNA-125b expands hematopoietic stem cells and enriches for the lymphoid-balanced and lymphoid-biased subsets," Proceedings of the National Academy of Sciences of the United States of America, vol. 107, no. 50, pp. 21505-21510, 2010.

[68] E. R. Lechman, B. Gentner, P. Van Galen et al., "Attenuation of miR-126 activity expands HSC in vivo without exhaustion," Cell Stem Cell, vol. 11, no. 6, pp. 799-811, 2012.

[69] E. Yildirim, J. E. Kirby, D. E. Brown et al., "Xist RNA is a potent suppressor of hematologic cancer in mice," Cell, vol. 152, no. 4, pp. 727-742, 2013.

[70] M. Jan, T. M. Snyder, M. R. Corces-Zimmerman et al., "Clonal evolution of preleukemic hematopoietic stem cells precedes human acute myeloid leukemia," Science Translational Medicine, vol. 4, no. 149, 2012.

[71] L. I. Shlush, S. Zandi, A. Mitchell et al., "Identification of pre-leukaemic haematopoietic stem cells in acute leukaemia," Nature, vol. 506, no. 7488, pp. 328-333, 2014.

[72] M. R. Corces-Zimmerman, W.-J. Hong, I. L. Weissman, B. C. Medeiros, and R. Majeti, "Preleukemic mutations in human acute myeloid leukemia affect epigenetic regulators and persist in remission," Proceedings of the National Academy of Sciences of the United States of America, vol. 111, no. 7, pp. 2548-2553, 2014.

[73] The Cancer Genome Atlas Research Network, "Genomic and epigenomic landscapes of adult de novo acute myeloid leukemia," The New England Journal of Medicine, vol. 368, pp. 2059-2074, 2013.

[74] B. Will, L. Zhou, T. O. Vogler et al., "Stem and progenitor cells in myelodysplastic syndromes show aberrant stage-specific expansion and harbor genetic and epigenetic alterations," Blood, vol. 120, no. 10, pp. 2076-2086, 2012.

[75] J.-P. J. Issa, “The myelodysplastic syndrome as a prototypical epigenetic disease," Blood, vol. 121, no. 19, pp. 3811-3817, 2013.

[76] R. Itzykson and P. Fenaux, "Epigenetics of myelodysplastic syndromes," Leukemia, vol. 28, no. 3, pp. 497-506, 2014.

[77] P. Wijermans, M. Lübbert, G. Verhoef et al., "Low-dose 5Aza-2'-deoxycytidine, a DNA hypomethylating agent, for the treatment of high-risk myelodysplastic syndrome: a multicenter phase II study in elderly patients," Journal of Clinical Oncology, vol. 18, no. 5, pp. 956-962, 2000.
[78] H. Kantarjian, Y. Oki, G. Garcia-Manero et al., "Results of a randomized study of 3 schedules of low-dose decitabine in higherrisk myelodysplastic syndrome and chronic myelomonocytic leukemia," Blood, vol. 109, pp. 52-57, 2007.

[79] H. Kantarjlan, J.-P. J. Issa, C. S. Rosenfeld et al., "Decitabine improves patient outcomes in myelodysplastic syndromes: results of a phase III randomized study," Cancer, vol. 106, no. 8, pp. 1794-1803, 2006.

[80] J. S. Fukumoto and P. L. Greenberg, "Management of patients with higher risk myelodysplastic syndromes," Critical Reviews in Oncology/Hematology, vol. 56, no. 2, pp. 179-192, 2005.

[81] A. P. Feinberg and B. Vogelstein, "Hypomethylation distinguishes genes of some human cancers from their normal counterparts," Nature, vol. 301, no. 5895, pp. 89-92, 1983.

[82] S. Maegawa, S. M. Gough, N. Watanabe-Okochi et al., "Agerelated epigenetic drift in the pathogenesis of MDS and AML," Genome Research, vol. 24, no. 4, pp. 580-591, 2014.

[83] G. Almanzar, S. Schwaiger, B. Jenewein et al., "Long-term cytomegalovirus infection leads to significant changes in the composition of the $\mathrm{CD}^{+} \mathrm{T}$-cell repertoire, which may be the basis for an imbalance in the cytokine production profile in elderly persons," Journal of Virology, vol. 79, no. 6, pp. 36753683, 2005.

[84] I. Kang, M. S. Hong, H. Nolasco et al., "Age-associated change in the frequency of memory $\mathrm{CD} 4^{+} \mathrm{T}$ cells impairs long term $\mathrm{CD} 4^{+}$ $\mathrm{T}$ cell responses to influenza vaccine," Journal of Immunology, vol. 173, no. 1, pp. 673-681, 2004.

[85] N.-P. Weng, "Aging of the immune system: how much can the adaptive immune system adapt?" Immunity, vol. 24, no. 5, pp. 495-499, 2006.

[86] J. F. Martin, S. D. Kristensen, A. Mathur, E. L. Grove, and F. A. Choudry, "The causal role of megakaryocyte-platelet hyperactivity in acute coronary syndromes," Nature Reviews Cardiology, vol. 9, no. 11, pp. 658-670, 2012.

[87] P. A. Stenberg, J. Levin, G. Baker, Y. Mok, and L. Corash, "Neuraminidase-induced thrombocytopenia in mice: effects on thrombopoiesis," Journal of Cellular Physiology, vol. 147, no. 1, pp. 7-16, 1991.

[88] A. Tomer, P. Friese, R. Conklin et al., "Flow cytometric analysis of megakaryocytes from patients with abnormal platelet counts," Blood, vol. 74, no. 2, pp. 594-601, 1989.

[89] L. Corash and J. Levin, "The relationship between megakaryocyte ploidy and platelet volume in normal and thrombocytopenic C3H mice," Experimental Hematology, vol. 18, no. 9, pp. 985-989, 1990.

[90] G. Lippi, T. Meschi, and L. Borghi, "Mean platelet volume increases with aging in a large population study," Thrombosis Research, vol. 129, no. 4, pp. e159-e160, 2012.

[91] I. Santimone, A. di Castelnuovo, A. de Curtis et al., "White blood cell count, sex and age are major determinants of heterogeneity of platelet indices in an adult general population: results from the MOLI-SANI project," Haematologica, vol. 96, no. 8, pp. 1180-1188, 2011.

[92] H. Raslova, A. Kauffmann, D. Sekkaï et al., "Interrelation between polyploidization and megakaryocyte differentiation: a gene profiling approach," Blood, vol. 109, no. 8, pp. 3225-3234, 2007.

[93] H. Huang and A. B. Cantor, "Common features of megakaryocytes and hematopoietic stem cells: what's the connection?" Journal of Cellular Biochemistry, vol. 107, no. 5, pp. 857-864, 2009. 
[94] F. J. de Sauvage, K. Carver-Moore, S.-M. Luoh et al., "Physiological regulation of early and late stages of megakaryocytopoiesis by thrombopoietin," Journal of Experimental Medicine, vol. 183, no. 2, pp. 651-656, 1996.

[95] H. Yoshihara, F. Arai, K. Hosokawa et al., “Thrombopoietin/MPL signaling regulates hematopoietic stem cell quiescence and interaction with the osteoblastic niche," Cell Stem Cell, vol. 1, no. 6, pp. 685-697, 2007.

[96] H. Qian, N. Buza-Vidas, C. D. Hyland et al., "Critical role of thrombopoietin in maintaining adult quiescent hematopoietic stem cells," Cell Stem Cell, vol. 1, no. 6, pp. 671-684, 2007.

[97] I. Bruns, D. Lucas, S. Pinho et al., "Megakaryocytes regulate hematopoietic stem cell quiescence through CXCL4 secretion," Nature Medicine, vol. 20, no. 11, pp. 1315-1320, 2014.

[98] M. Zhao, J. M. Perry, H. Marshall et al., "Megakaryocytes maintain homeostatic quiescence and promote post-injury regeneration of hematopoietic stem cells," Nature Medicine, vol. 20, no. 11, pp. 1321-1326, 2014.

[99] C. A. de Graaf, M. Kauppi, T. Baldwin et al., "Regulation of hematopoietic stem cells by their mature progeny," Proceedings of the National Academy of Sciences of the United States of America, vol. 107, no. 50, pp. 21689-21694, 2010.

[100] P. Deloukas, S. Kanoni, C. Willenborg et al., "Large-scale association analysis identifies new risk loci for coronary artery disease," Nature Genetics, vol. 45, no. 1, pp. 25-33, 2013. 

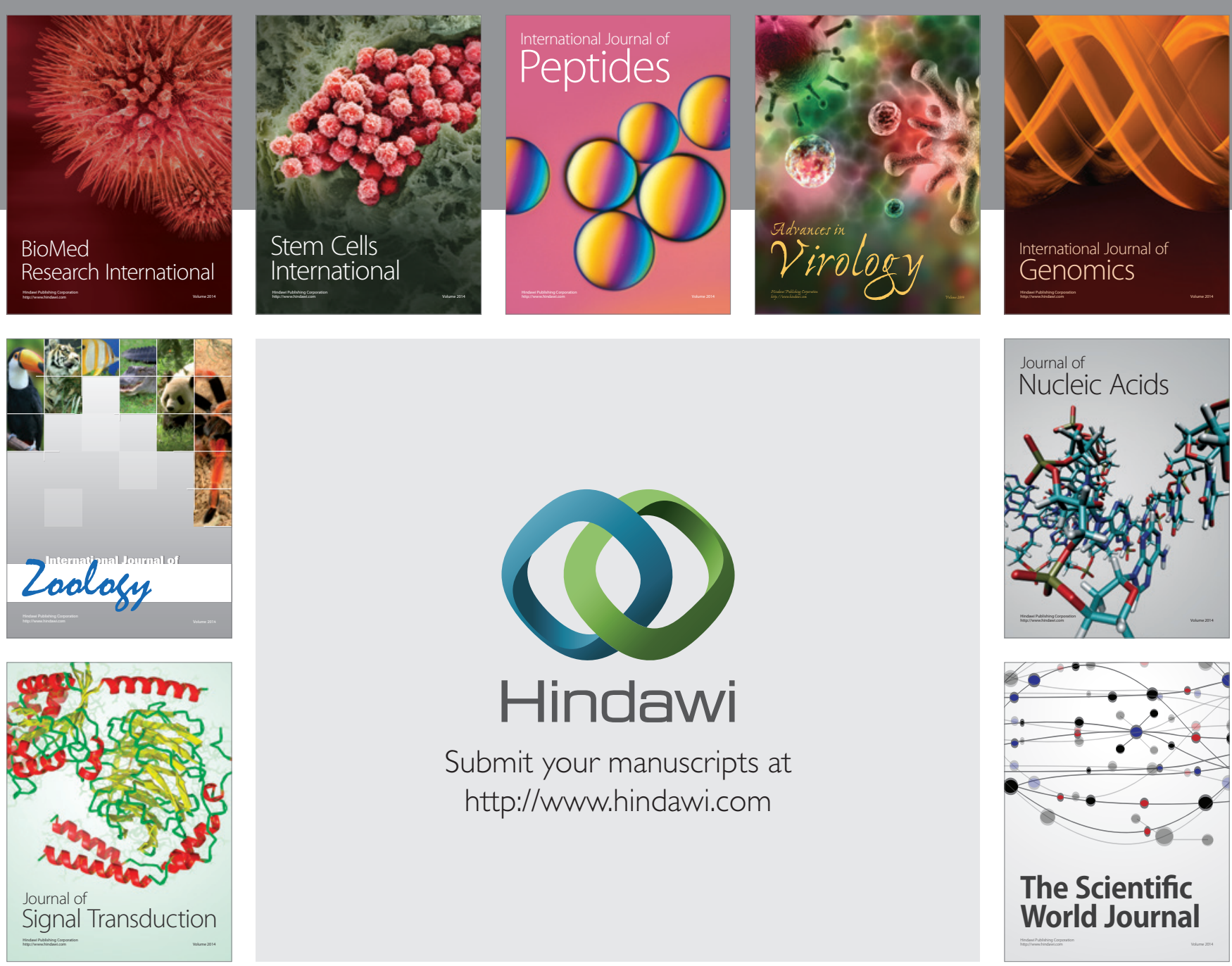

Submit your manuscripts at

http://www.hindawi.com
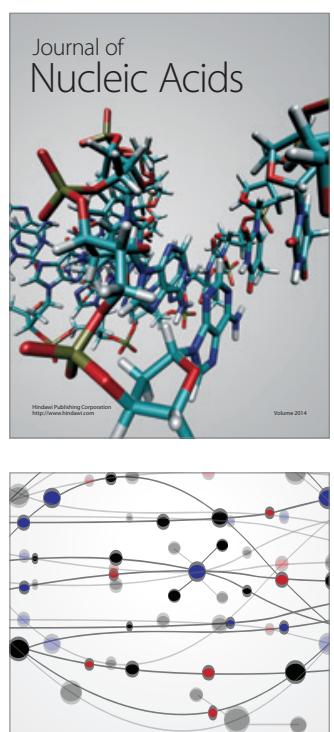

The Scientific World Journal
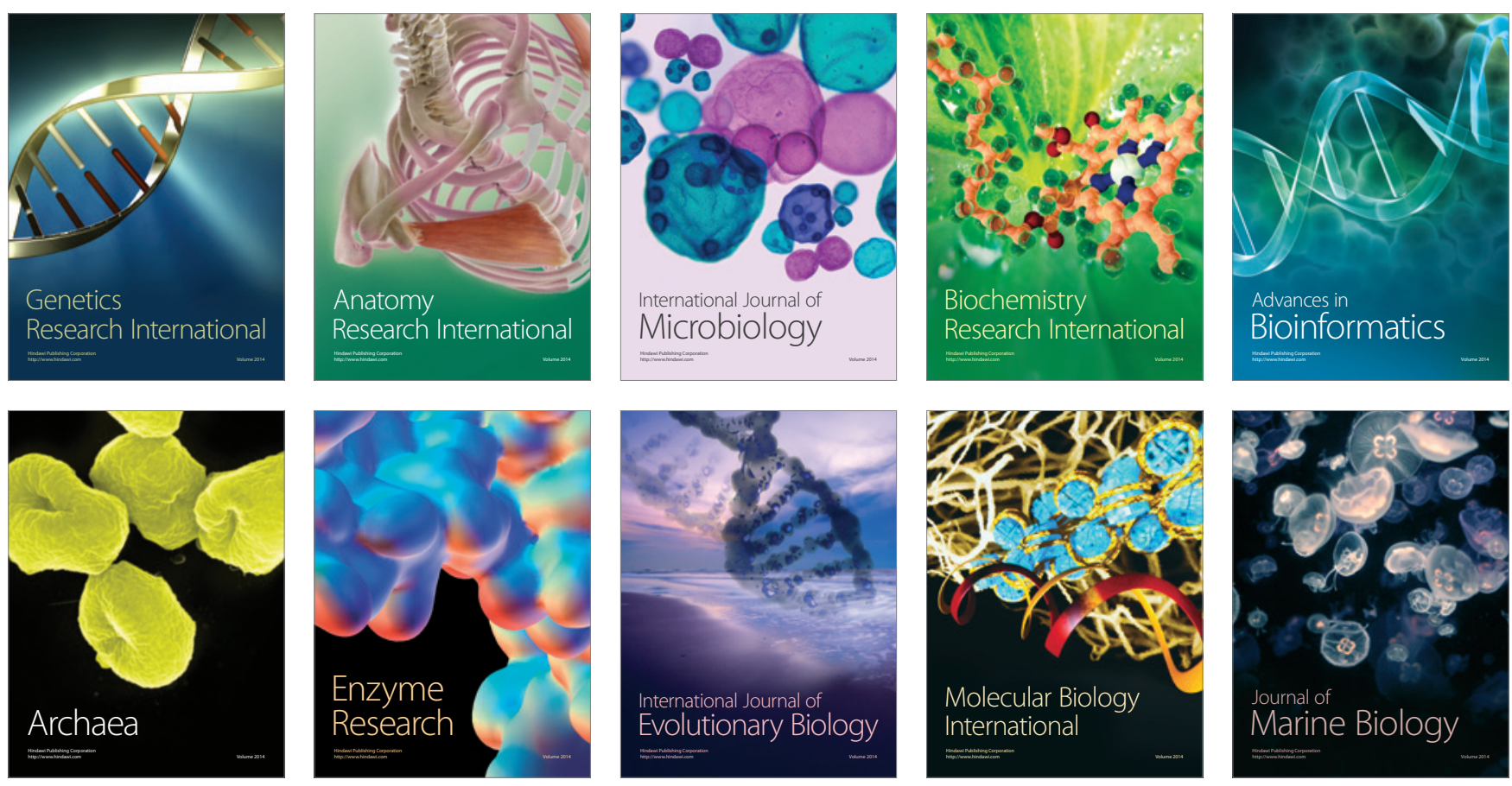